\title{
Modelos de negocio para el periodismo deportivo de nicho en el contexto postindustrial
}

institucional.us.es/ambitos/

\author{
José Luis Rojas Torrijos \\ Universidad de Sevilla \\ jlrojas@us.es
}

\section{Cristian Ramón Marín Sanchiz}

Universidad Miguel Hernández

cristian.marin@alu.umh.es

English Version: For business models sports journalism niche in the context postindustrial.

\begin{abstract}
Resumen
Este estudio pretende ahondar en los cambios que la llegada del ecosistema digital ha supuesto para el negocio de los medios de comunicación deportivos, que en muchos casos han apostado por el nicho para generar valor añadido a través de la hiperlocalización o hiperespecialización o la creación de una imagen de marca que permita al usuario diferenciarlo de competidores. Tomando como

muestra el censo de nuevos medios lanzados por periodistas desde 2008 en

España, se analizan aquellos emprendimientos deportivos de nicho cuyas estrategias han resultado más sostenibles y rentables para hallar patrones de

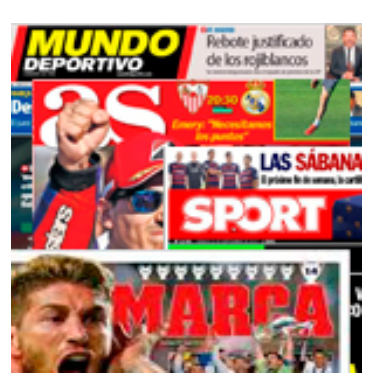
éxito que permitan extrapolar su experiencia a otras iniciativas emprendedoras.
\end{abstract}

\section{Palabras clave}

Periodismo deportivo, periodismo digital, emprendimiento, innovación, periodismo de nicho.

\begin{abstract}
This study aims to analyse changes produced by the advent of digital ecosystem for sports media business, many of which targeted towards niches in order to generate added value through hyperlocal or hyperspecialed content or creation of a brand that allows a user to distinguish it from competitors. Taking

the census of new media launched by journalists since 2008 in Spain, we look into those sports niche journalism outlets whose strategies have been more sustainable and profitable so as to find patterns of success and extrapolate their experience to other entrepreneurship news projects.
\end{abstract}

Keywords Sports journalism, digital journalism, entrepreneurship, innovation, niche journalism.

\section{ÁMBITOS \\ 2018 \\ $n^{2} 3 B$}

\section{INTRODUCCIÓN}

El desarrollo de internet ha supuesto un verdadero reto para la viabilidad de las empresas periodísticas que no solo han visto modificada las maneras de producir y distribuir sus contenidos, sino sobre todo el funcionamiento de las estructuras de toda una industria. Anderson, Bell y Shirky aseguraron en su obra Periodismo postindustrial: adaptación al presente que uno de los problemas de la prensa actual es que, "como en cualquier revolución, lo viejo se rompe mucho antes de que se construya lo nuevo" $(2013,57)$. El descenso de la inversión en publicidad que trajo consigo la crisis económica mermó las cuentas de 
pérdidas y ganancias de una buena parte de los medios de comunicación en España, pero con el auge del ámbito digital y de las nuevas formas de uso del papel han nacido diversas iniciativas emprendedoras que han alcanzado la sostenibilidad gracias a la diversificación de su actividad y de sus vías de ingresos, pero también a la explotación de nichos informativos.

La realidad actual es que, en un acelerado y forzoso proceso de reconversión hacia lo digital, la gran mayoría de las organizaciones de noticias todavía no han sabido adaptarse al nuevo ecosistema mediático, en parte porque siguen considerando la innovación más como una solución a corto plazo, ligada a la mera aplicación de las tecnologías para distribuir contenidos y dar respuesta a los cambios repentinos de consumo de la información por parte de los usuarios, que como una estrategia con una perspectiva largoplacista en la que se busquen maneras de cambiar el comportamiento de los consumidores (Mann, 2015).

Sobre esta cuestión, Phillips señala como un error por parte de muchos medios el hecho de no haber tenido más en consideración que la disrupción digital iba más allá del modelo informativo. A su juicio, los medios innovaron sus productos (unos mejor que otros), pero sus innovaciones no trajeron consigo los cambios profundos que necesitaban sus organizaciones porque los directores de estas empresas no tuvieron en cuenta cómo los innovadores atacarían. "Si hubieran innovado sus modelos de negocio a la misma velocidad que su periodismo, podrían haber salido adelante de una mejor manera" $(2014,108)$.

En opinión de Casero-Ripollés (2012), las empresas periodísticas han evidenciado una incapacidad para instaurar una fórmula viable para obtener recursos económicos de su actividad digital, hasta el punto de provocar incluso una paradoja: "el consumo de noticias online aumenta de forma notable, pero los editores de prensa no consiguen traducir ese incremento de la demanda en ingresos financieros que hagan rentable su negocio. Es decir, no logran monetizar la ampliación de la audiencia".

Algo más optimista se muestra Jeff Jarvis, quien en el prólogo de la obra Entrepreneurial Journalism (Briggs, 2012: XV-XVII), considera que los nuevos medios pueden dar pistas a los grandes a la hora de afrontar la disrupción digital, haciéndolo a partir de dos supuestos: la creación de nuevos modelos de negocio para emprendimientos "que no serán como los gigantes mediáticos del pasado, sino mucho más pequeños, pero sus costes serán bajos, por lo que podrán obtener beneficios a corto plazo»; y repensar la verdadera naturaleza de las noticias y su valor para el público: "debemos replantear la relación con nuestra audiencia colaborando con ella porque ahora podemos hacerlo así»».

A este respecto, el propio Mark Briggs (2012, XX) destaca cómo el nuevo ecosistema informativo se ha convertido en "un campo de oportunidades para los periodistas emprendedores», que pueden aprovechar el hecho de que las características que impulsan el éxito en periodismo también valen para hacer negocios: "Los periodistas saben cómo hacer buenas preguntas y encontrar fuentes de información solventes, cómo buscar agujeros y maniobrar para llenarlos, cómo navegar frente a adversidades y hallar una nueva ruta, cómo no ser engañados por el ruido. Sobre todo, los periodistas saben cómo reunir información y producir buenos contenidos para satisfacer las necesidades de la gente».

En este contexto de cambio y oportunidades, los nuevos emprendimientos y proyectos periodísticos promovidos por profesionales que han surgido en los últimos años en España aún necesitan tiempo para consolidarse a partir de unas estructuras económicas que sean mucho más estables y que les permitan hacer un periodismo independiente usando nuevos modelos de negocio que no estén tan supeditados a una publicidad tradicional que escasea, que diversifiquen sus vías de ingresos y que replanteen su relación con la audiencia a la que se dirigen para hacerla más partícipe de los contenidos (Newman, 2016).

Así, la Asociación de la Prensa de Madrid en su último Informe anual de la profesión periodística señala que desde 2008 han surgido en España 579 nuevos medios, de los que 458 siguen funcionando y 121 cerraron o interrumpieron su actividad. Además, observa que poco a poco muchos de ellos empiezan a asentarse. Según una encuesta realizada a promotores y responsables de estos emprendimientos periodísticos, se indica que "no hay duda de que este incipiente segmento de los medios de comunicación está cosechando 
un éxito, aunque sea relativo y acorde con las difíciles circunstancias que atraviesa el sector y la profesión", si bien el estudio agrega que "la permanencia de estos proyectos en el tiempo no se debe solo a un posible y relativo éxito empresarial; la dureza y persistencia de la crisis económica, con la falta de alternativas que conlleva, tiene mucho que ver al respecto" $(2015,78)$.

También está llamada a desempeñar un papel crucial en la sostenibilidad de estas nuevas empresas periodísticas su capacidad para innovar tanto en la confección de sus productos y servicios como en el uso de las nuevas tecnologías y también en los procesos de producción y distribución de los contenidos y en las maneras de comercializarlos. Uno de los estudios más destacados en este campo es el Ranking de Innovación Periodística que elabora anualmente la Universidad Miguel Hernández y que, entre los resultados que arroja, indica que "la innovación y la experimentación en el panorama periodístico nacen más y mejor fuera de la industria mediática tradicional" (Carvajal, 2014).

\subsection{La apuesta por el nicho en la información deportiva}

En ese camino hacia la necesaria estabilidad de sus estructuras financieras en el nuevo orden mediático digital, muchos nuevos medios dedicados a la información deportiva han apostado por la superespecialización para hacerse un hueco en el mercado y atender a una audiencia real y potencial que demandaba otro tipo de productos y servicios que hasta ahora no estaban siendo suficientemente atendidos en un mercado donde se consume mucha información deportiva pero con escasa diversidad temática.

De hecho, recientes investigaciones como la desarrollada por Ignacio Calvo (2016) apuntan a la "sobreinformación" sobre fútbol, que copa más del 92\% del total de las informaciones de portada de los diarios deportivos digitales de referencia en España (Marca, As, Sport y Mundo Deportivo), al mismo tiempo que al "silencio informativo" o infravaloración, tanto en cantidad como en jerarquización, con respecto al resto de modalidades, que tienen una presencia residual del $7,88 \%$ del total de los contenidos más destacados en la web.

De esta forma, la creación de valor añadido para el usuario, detectando primero cuáles son sus necesidades informativas reales y luego aplicando una solución que las cubra de la forma más original, atractiva y solvente que sea posible, se ha convertido en una condición indispensable para el funcionamiento de los medios deportivos de nicho, partiendo de la premisa de que "el enfoque en productos y mercados saturados reduce las posibilidades de éxito de una iniciativa periodística" (Manfredi, Rojas y Herranz, 2015b).

Esa propuesta de valor es, precisamente, el eje que articula los modelos de negocio de este tipo de proyectos, aunque no es el único cambio que hay que tener en cuenta. También está, como señalábamos antes, la estrategia de financiación. En el modelo industrial, los medios de comunicación deportivos vivían de la publicidad pero, a partir de 2008, internet era el único ámbito que presentaba datos positivos en este aspecto.

En la actualidad, aunque la inversión en publicidad aumentó un 7\% durante el primer semestre de 2015 respecto al mismo período de 2014 (Infoadex, 2015), las iniciativas deportivas de nicho han modificado sus modelos de negocio para poner la creación de valor en el centro del proceso y, además, han diversificado las vías de ingresos (tienda con productos exclusivos, comunidad de lectores, contenidos premium por suscripción, alianzas con otros medios, servicios de asesoría o patrocinios, entre otros).

Lo cierto es que la información deportiva se ha convertido en la punta de lanza de la ola emprendedora que vive actualmente el periodismo español. Así, de los 458 nuevos proyectos periodísticos lanzados desde enero de 2008 y que continúan en activo, 41 se dedican a la información deportiva, según la Asociación de la Prensa de Madrid (2015). Aunque aquí se señala que el 9,1\% son de temática deportiva, según los datos del blog Periodismo Deportivo de Calidad [1], este porcentaje se eleva en realidad a casi el 20\% con más de 70 nuevos medios deportivos de toda índole. 
Entre esas iniciativas se puede encontrar una gran diversidad de temáticas y soportes: desde revistas en papel especializadas en ciclismo hasta portales que ofrecen información sobre la Premier League, pasando por revistas interactivas especializadas en motociclismo. Según los estudios desarrollados en este campo por Manfredi, Rojas y Herranz (2015), se observan dos claras tendencias que refuerzan la idea de nicho de audiencia (información hiperlocal y segmentación temática por deportes), así como nuevas maneras de gestionar los contenidos orientadas a internet y cada vez más vinculadas a la marca personal del periodista, y nuevas maneras de presentar los contenidos a partir del replanteamiento de géneros y formatos y, sobre todo, del uso creciente de las nuevas narrativas digitales.

La diversidad de estos proyectos y lo reciente del fenómeno del emprendimiento periodístico en España han impedido que exista un conocimiento profundo sobre las estrategias que les han permitido alcanzar la sostenibilidad o los motivos por los que, aquellos que cerraron, no fueron rentables. En este sentido, la presente investigación pretende satisfacer un interés sectorial, que pasa por ofrecer a los propietarios de nuevos medios de comunicación pistas para lograr un modelo de negocio rentable, sostenible y escalable. Esta vertiente profesional se complementa con la resolución de un interés académico, cuyo principal reto es analizar las propuestas de negocio que, en el mercado español, ponen sobre la mesa los pure players del ámbito deportivo.

Por otra parte, este estudio busca dar respuesta a un interés económico que reside en la necesidad de un modelo sostenible para la profesión periodística. El emprendimiento supone una alternativa a las formas tradicionales de empleo y es imprescindible analizar aquellos casos de éxito, para así poder aproximarse a la formulación de una serie de pautas que faciliten la creación y el mantenimiento de nuevos proyectos periodísticos.

\section{ESTUDIO DE CASOS}

\subsection{Hipótesis}

Los modelos de negocio de los medios de comunicación especializados en periodismo deportivo de nicho tienen como eje central la generación de valor añadido para el usuario y su éxito depende de, entre otros, cuatro componentes. El primero de ellos es la hiperespecialización o hiperlocalización (Bradshaw, 2012), que permite satisfacer necesidades concretas de los usuarios y aprovechar las posibilidades de la larga cola.

El segundo componente es la diversificación de fuentes de financiación, entre las que destacan la publicidad, el cobro por contenidos, la venta de tecnología y datos, los eventos, el freelancing y la consultoría (Sirkkunnen, Cook, 2012). En tercer lugar, hay que atender a la importancia de crear una imagen de marca que permita al usuario diferenciar el medio de sus competidores (Bruno y Nielsen, 2012). Y, por último, esta hipótesis contempla que la contención de costes es un hecho determinante para el éxito de los medios deportivos de nicho en el contexto postindustrial (Bruno y Nielsen, 2012).

\subsection{Objetivos}

El principal objetivo de esta investigación es analizar los modelos de negocio de tres medios de comunicación españoles que sean sostenibles y que operen en el mercado de la información deportiva de nicho, para así encontrar patrones y elementos de éxito que permitan extrapolar su experiencia a otras iniciativas.

En segundo lugar, el texto pretende conocer nuevas tendencias en lo relativo a modelos de negocio para la prensa deportiva postindustrial española, así como aquellos elementos que favorecen el éxito de un determinado modelo, como pudieran ser la generación de una comunidad, la especialización o la creación de redes de colaboración. 
El tercer objetivo marcado pasa por descubrir el papel que tiene la publicidad en el mercado actual. Esta fuente de ingresos, que durante años fue el pilar sobre el que se sostenía el periodismo deportivo, ha sufrido numerosos cambios y modificaciones desde que nacieron los pure players, y por esta razón se pretende obtener una fotografía de la situación actual de la publicidad y compararla con la del resto de fuentes de ingresos.

Por último, esta investigación permitirá conocer si los medios de comunicación sostenibles, con más impacto a nivel de visitas un mayor número de trabajadores han apostado por innovar en sus modelos de negocio.

\subsection{Metodología}

La presente investigación emplea una metodología basada en el estudio de casos dividido en dos fases. La primera abarca la recopilación de casos y la selección de la muestra, mientras que en la segunda se desarrolla un análisis cualitativo de los datos obtenidos y se elabora la valoración final.

\subsubsection{Primera fase: selección de la muestra}

El punto de partida de esta fase es el Censo de nuevos medios deportivos lanzados por periodistas en España (Rojas Torrijos, 2014), que recoge aquellos pure players españoles que están centrados en el ámbito deportivo. Esa muestra inicial se sometió a un cribado que permita discriminar los medios con más lectores a través de la siguiente fórmula:

Valor $=($ Ranking Alexa*0’5) $+($ Ranking similarweb*0,5) $(1)$

Los diez medios más valorados se sometieron, posteriormente, a un segundo filtro que tenía por objetivo seleccionar aquellas iniciativas que cumpliera el requisito de la rentabilidad, siguiendo la línea de investigaciones anteriores relacionadas con la investigación de modelos de negocio a partir del estudio de casos (Sirkkunen, Cook, Pekkala, 2012).

El filtrado se llevó a cabo mediante un cuestionario cerrado con el que se conoce y puntúa:

- Si el medio es rentable (3 puntos).

- Desde hace cuánto tiempo, en el caso de que lo sea (1 punto por cada año o 0,5 por fracción).

- Cuántas personas trabajan y de qué forma (tiempo parcial, colaboradores...) (1,5 puntos por trabajador a tiempo completo, 1 por trabajador a tiempo parcial y 0,25 por colaboradores).

\subsubsection{Segunda fase: análisis de modelos de negocio}

Esta segunda fase se divide, a su vez, en dos apartados: el heurístico o de descubrimiento y el de confirmación (Martínez Carazo, 2012). El primero, que tiene como objetivo describir los modelos de negocio estudiados, se centró en los tres medios de comunicación con una mayor puntuación en la primera fase. El segundo pretende comprobar si los datos obtenidos confirman la hipótesis que dio lugar a la presente investigación.

\subsubsection{Fase heurística}

Para conocer con detalle sus respectivos modelos de negocio se empleó, en primer lugar, una entrevista que incluía una serie de preguntas comunes a todas las iniciativas más una serie de cuestiones que permitieran descubrir y comprender las particularidades de cada caso.

La relación de preguntas para el cuestionario abierto fue la siguiente:

.¿Cuáles son las fuentes de ingresos? 
• ¿Cuál es la estructura de costes?

- Perfiles de los empleados y por qué

• ¿Cuál es la proporción de costes-ingresos que tiene el medio?

. ¿Qué porcentaje del total de ingresos supone cada uno?

- ¿Son diferentes a las iniciales del proyecto?

- ¿Cuáles se han añadido y/o rechazado? ¿Por qué?

.¿Qué diferencia su modelo de negocio del de sus competidores?

. ¿Qué factor o factores permiten que el modelo de negocio funcione?

. ¿Tener una imagen de marca fuerte es imprescindible para que funcione un medio deportivo de nicho?

.¿Qué valor añadido ofrece su medio a los lectores?

- ¿Hasta qué punto podría crecer la empresa con el modelo de negocio actual?

- ¿Qué cambiaría de él?

. ¿Se han planteado alguna innovación en este sentido?

. ¿En qué medida su modelo de negocio favorece el crecimiento?

. ¿El modelo de negocio es escalable?

• ¿Cuál es el objetivo del modelo de negocio? (subsistir, crecer, tener beneficios, especular...)

Para asegurar la validez de los datos, así como una correcta interpretación de la información cualitativa obtenida en la entrevista, se recurre a las estrategias de triangulación (Stake, 1995). Si la primera fase de esta metodología permitía eliminar el componente de la arbitrariedad a la hora de seleccionar los casos que serán analizados, la triangulación permite reforzar las conclusiones obtenidas al analizar las respuestas.

Esta validación se llevó a cabo mediante una triangulación de métodos (Arias, 1999) que utilizaba un cuestionario cerrado para concretar diversos aspectos (porcentajes de crecimiento o decrecimiento de los ingresos desde que nacieron, variaciones concretas en el modelo de negocio...) con las que aportar un contexto más comprensible y delimitado a las respuestas de la entrevista inicial.

Para completar la triangulación, se entrevistó a, al menos, dos responsables de cada medio en aquellos casos que fue posible. De este modo, se podían contrastar respuestas, reforzar los puntos comunes y encontrar posibles discordancias que necesitaran ser resueltas.

\subsubsection{Fase de confirmación}

En este último punto se comprobó si los resultados obtenidos confirman la hipótesis planteada y se analizaron aquellos elementos discordantes en cada uno de los casos estudiados. Del mismo modo, se buscaron patrones en aquellos puntos que no confirmaban la hipótesis para encontrar las posibles causas o alternativas desarrolladas por los medios de comunicación que conforman la muestra de la presente investigación.

\section{RESULTADOS}


Los diez medios que pasaron la primera fase, que tenía por objetivo seleccionar la muestra que se trabajaría posteriormente, fueron los siguientes: Vavel, Sportyou, El Desmarque, Todocircuito.com, kiaenzona.com, Diariogol, Ecosdelbalon, Motorbike Magazine, Spherasports, Martiperarnau.com.

A partir de esta relación de proyectos, lo primero que se hizo fue atender al criterio objetivo planteado en la metodología y descartar aquellos proyectos que no se ajustaran, dentro de unos mínimos, a la rigurosidad periodística. En este caso, la única iniciativa que no pasó ese filtro fue Diariogol. Con la muestra pulida, se contactó con los responsables, con el objetivo de recopilar la información necesaria para llevar a cabo el segundo filtro.

Sin embargo, hubo una falta de respuesta generalizada, y también un caso en el que el contrato con una lanzadera de empresas no permitía ofrecer información sobre el modelo de negocio del medio. Por esta razón, se decidió atender a aquellos de los que se disponía de más información: El Desmarque y Todocircuito.com, dos medios que atienden nichos completamente distintos pero que, al mismo tiempo, comparten una apuesta firme por el ámbito digital. Tras realizar el cuestionario, revisar las respuestas y confirmar aquellos puntos en los que existían dudas que dieran lugar a la interpretación, se obtuvieron los siguientes resultados:

\subsection{Fuentes de ingresos}

Tanto El Desmarque como Todocircuito.com se financian, casi íntegramente, por publicidad. En el caso del medio especializado en fútbol, esta fuente de ingresos supone el $100 \%$, mientras que en el del medio sobre motociclismo, el 95\%. La publicidad en los dos proyectos toma distintas formas. Desde los clásicos banners hasta formatos más recientes, como el branded content, los custom ads o promociones en sitios externos, como las Redes Sociales. Todocircuito.com recibe un $5 \%$ de sus ingresos totales del Club Todocircuito, que ofrece una serie de descuentos y ventajas al socio a cambio de una cuota anual.

Las fuentes iniciales y actuales no han sufrido grandes modificaciones. En El Desmarque han rechazado aquellas campañas de publicidad que buscan el pago por rendimiento. "Si alguien quiere que El Desmarque promueva su producto y solo quiere ir a «éxito», no puede trabajar con nosotros. Estamos dispuestos a tener modelos con variables para el cliente final en función de rendimiento de la publicidad (que venda más, vamos), pero el $100 \%$ del modelo no puede estar basado en performance (CPA, por ejemplo), porque no controlamos la calidad del producto ofertado, sea éste digital o físico", explica Javier Padilla.

Otra de las vías que se descartaron son aquellas que canibalizaba el propio medio. En este caso, coincide también con Todocircuito.com, puesto que se experimentó con la inclusión de un outlet de productos que ofreciera ingresos a través de comisiones, pero los responsables del proyecto se dieron cuenta de que existía un problema: "No nos conviene vender material porque muchos de nuestros anunciantes son, precisamente, tiendas", asegura Alfonso Somoza, CEO del medio. Además, "los beneficios eran muy bajos".

De acuerdo con Padilla, el éxito de un medio de comunicación basado en la publicidad, que está obligado a competir en escala, pasa por optimizar los procesos comerciales, puesto que la buena progresión financiera de El Desmarque se debe a un factor clave: "Ahora sabemos vender publicidad y antes no".

\subsection{Creación de valor añadido}

De acuerdo con la información proporcionada por el responsable de Todocircuito.com, el factor que posibilitó el éxito de este medio fue "ofrecer un contenido que antes no estaba disponible en la Red (un calendario que recopilaba todos los eventos organizados en los circuitos españoles). De ese modo, miles de motoristas podían consultar y planificar sus rodadas en un mismo sitio, sin tener que perderse entre decenas de webs". Pero no fue el único: "Somos muy cercanos al usuario. Cualquier persona puede escribir a cualquier miembro del staff por distintos medios (foro, mensaje privado, e-mail, Facebook, Twitter) y recibirá respuesta casi inmediata". 
En el caso de El Desmarque, el valor añadido pasa por darle la misma importancia a los equipos locales "que al Real Madrid en Marca", aunque parte del éxito, según Padilla, es haber creado un «parque temático» del fútbol: “Encuentras información del Betis, pero también la información de puntuación para Comunio, la posibilidad de apostar, vídeos, etcétera. Y en ese sentido avanzaremos".

Los dos periodistas coinciden en que es "absolutamente imprescindible" generar un valor añadido para el usuario. "Los 10 años que llevo trabajando en internet me han dejado claro que sólo hay dos formas de conseguir que un negocio prospere en la Red: solucionar una necesidad ofreciendo un producto novedoso nunca visto, o bien contar con un generoso presupuesto en publicidad para el lanzamiento", concluye Somoza.

Respecto a la necesidad de que los trabajadores sean conscientes de la importancia del valor añadido para los usuarios, ambos periodistas coinciden. "Tienen que serlo, porque es la base de la empresa. Sin ese valor añadido no habría diferencia con otros medios", reconoce Somoza. Padilla, por su parte, defiende que sus empleados son parte del proceso periodístico: “Ellos saben que deben estar con nosotros tanto en la creación de contenidos como en la distribución o en la amplificación de campañas de publicidad. Su rol editorial es uno y el de ayuda a la monetización es otro."

\subsection{Estructura y contención de costes}

La estructura de costes de Todocircuito.comestácompuesta por el personal, el alquiler de la oficina, los dos servidores donde se aloja la web, la cuota mensual del servicio de mailing, los gastos de gestoría y los gasto en combustibles y alojamiento para viajes y pruebas. En su caso, los gastos suponen un $85 \%$ de los ingresos mensuales. Con los beneficios se crea un “colchón” económico para la empresa, que se reinvierte en mejoras tecnológicas.

En El Desmarque, este apartado está compuesto por el personal y oficinas (60\%), infraestructura tecnológica (servidores, software... que suponen el 20\%), formación (10\%) y marketing en redes sociales (10\%). La proporción costes-ingresos es de, aproximadamente, un 70-100.

Además de la similitud en las principales partidas, que abarcan personal, oficinas y tecnología, destaca que la diferencia entre el modelo de negocio de estos dos medios y el de sus competidores, es, precisamente, la contención de costes. Según Alfonso Somoza, “otros portales y medios cuentan con plantillas que multiplican por cuatro y por cinco" a la de su medio, "sin llegar en ocasiones a igualar el tráfico". Esto, sin embargo, tiene un aspecto negativo: "También es cierto que, al no tener muchos trabajadores, desatendemos áreas como la comercial, donde podríamos mejorar mucho más si tuviésemos personal dedicado a ello".

De acuerdo con Javier Padilla, esa distinción pasa porque los costes de producción “son más bajos y adecuados a Internet". Incluso están experimentando con nuevas vías: "Empezamos a sacar dinero de la parte de datos, pero es un modelo aún incipiente".

\subsection{Escalabilidad}

Padilla y Somoza coinciden en que los modelos de negocio de sus respectivos proyectos son escalables, porque permiten que la audiencia y los ingresos crezcan exponencialmente sin que, necesariamente, se disparen los costes de producción.

No obstante, Padilla advierte de los potenciales problemas: “¿La escalabilidad puede tener un techo y llegar a tener mucho 'espacio invendido'? Sí, podría ocurrir si no somos capaces de ofrecer rendimiento a las marcas que confían en nosotros. Pero en ese sentido estamos preparados para cambiar el modelo y probar nuevos formatos de monetización con cierta rapidez. La estructura es muy flexible." 
Con el modelo actual, sería "complicado" que Todocircuito.com crezca "mucho más". La clave, en todo caso, sería "realizar una inversión medianamente fuerte en busca de dos objetivos: el primero, incrementar la plantilla a nivel de redacción y comercial, y por otro lado, expandir la temática de la web, bien saltando a otros países o cubriendo otras temáticas en España". El Desmarque, en cambio, podría crecer "mucho".

\subsection{Imagen de marca}

La visión sobre la importancia que tiene generar una imagen de marca fuerte es distinta. De acuerdo con Padilla, no es imprescindible porque "Internet se mueve por contenidos de calidad y virales". "Por eso existen fenómenos como los youtubers. Primero es el contenido y, después, la creación de la marca vinculada a estos. Las primeras 10 veces que alguien veía un contenido de El Rubius ni siquiera se fijaba en quién era ese chaval que hacía el payaso jugando a la consola. Ahora ya lo buscan por su nombre. En el caso de El Desmarque, nos ocurría en algunas delegaciones que nos conocían por algunas exclusivas en vídeo o audio, pero no nos ponían «cara» en cuanto a marca", detalla. En lo referente a la inversión, considera que "es más importante invertirlo en crear buenos contenidos que lleven la marca adosada de alguna manera. Dicho de otra forma: branded content de ti mismo como fórmula de crecimiento".

Para Somoza, en cambio, sí que es condición necesaria, debido a que "blogs y webs de motos hay cientos en la red. Si tu imagen de marca es reconocible, ayuda mucho a asomar la cabeza".

\subsection{Factores de éxito, objetivos e innovación}

El principal factor que determina el buen funcionamiento del modelo de negocio de El Desmarque es "el volumen de los consumidores que se consigan para cada nuevo producto", según Padilla. Por esta razón, el medio sigue la metodología Lean Startup (2): “Creamos productos, los lanzamos rápido y los medimos. Aquello que funciona se potencia y, lo que no, se cierra. El apego por una idea errónea te puede matar en Internet muy rápidamente".

Para Todocircuito.com, el éxito reside en "la confianza tanto de grandes anunciantes como de tiendas y negocios más modestos". Esta diversificación de clientes permite que los ingresos "incrementan año tras año", aunque la progresión de la empresa se tome "siempre con cabeza y sin correr riesgos que pongan en peligro la empresa".

El objetivo del medio especializado en motociclismo es, por tanto, "generar beneficios, con un ojo puesto en el crecimiento". La meta para el Desmarque también es tener beneficios. No buscan subsistir, ni tampoco crecer o especular.

En este contexto, Javier Padilla no cambiaría el modelo de negocio de El Desmarque, puesto que "funciona bien". A pesar de ello, sí que se han planteado alguna innovación, como "la orientación a vídeo como soporte principal del contenido". A Alfonso Somoza le gustaría ampliar la plantilla, y también se han planteado algunas innovaciones. No concreta cuáles y, además, asegura que "la relación de ingresos/costes" no les permitiría introducirla.

\section{CONCLUSIONES}

Los resultados obtenidos en el presente estudio nos permiten confirmar la hipótesis de punto de partida: la generación de valor añadido es un requisito imprescindible para que un medio de comunicación deportivo sea rentable en el contexto postindustrial. No obstante, existen matices importantes respecto a los factores de éxito del modelo de negocio. De esta forma, llegamos a las siguientes conclusiones:

1. La hiperespecialización permite que los medios digitales especializados en información deportiva exploten un nicho de información y lo rentabilicen gracias a la creación de contenidos de interés para un público definido, ya sea a nivel de usuarios (lectores) o clientes (anunciantes). 
2. Asimismo, la generación de un valor añadido para los usuarios es una condición necesaria para el funcionamiento de un modelo de negocio periodístico en el contexto digital. Esta diferenciación se puede abarcar desde diversos ejes: desde la creación de contenidos exclusivos con un gran interés, pasando por ofrecer una cobertura y un enfoque únicos, e incluso el lanzamiento de productos tecnológicos.

3. La diversificación de fuentes de financiación no es una condición necesaria para el funcionamiento de un medio de comunicación deportivo en el contexto postindustrial. A pesar de que los dos medios estudiados están experimentando con nuevas vías de ingresos, únicamente la publicidad genera beneficios. En su caso, la clave reside en la optimización de los procesos de venta.

4. La contención de costes es una estrategia que ayuda a los medios deportivos de nicho a funcionar en el contexto digital. El personal es la partida de gastos que más recursos necesita, y en este contexto, el reto es conseguir una plantilla equilibrada, que no implique gastos superfluos y, al mismo tiempo, permita atender áreas como la comercial.

5. La metodología Lean Startup (orientada al lanzamiento de productos y el desarrollo de negocios, se basa en el aprendizaje validado, la experimentación científica y el ensayo-error) permite a los medios experimentar nuevos productos, contenidos y vías de ingresos de una forma económica y sin dedicar más recursos de los necesarios.

6. El modelo de negocio basado en publicidad para los medios especializados en el ámbito digital es escalable: es posible incrementar exponencialmente el número de usuarios ( $y$, por extensión, los ingresos) sin que sea necesario un aumento en los costes, más allá de algunos requisitos tecnológicos, como los servidores.

7. Entre las fuentes de ingresos que los medios han descargado, destacan aquellas que fagocitan otras vías, como pueden ser las ventas de productos deportivos o la venta de espacio publicitario para percibir un ingreso en función de rendimientos.

\section{BIBLIOGRAFÍA Y WEBGRAFÍA}

ANDERSON, C.W. BELL, E. y SHIRKY, C. (2013): Periodismo postindustrial: adaptación al presente. Huesca: Asociación de Periodistas de Aragón

http://www.ecicero.es/wp-content/uploads/2013/03/periodismo_postindustrial.pdf

APM (2015). Informe anual de la profesión periodística. Madrid: APM

ARIAS VALENCIA, M.M. (1999): La triangulación metodológica: sus principios, alcances y limitaciones. México: Universidad de Antioquia

http://www.uv.mx/mie/files/2012/10/Triangulacionmetodologica.pdf

BRADSHAW P. (2012) Disengaging from the news and hyperlocal engagement. Online Journalism Blog

http://ciruelo.uninorte.edu.co/pdf/pensamiento_gestion/20/5_El_metodo_de_estudio_de_caso.pdf

BRUNO, N., NIELSEN, R.K. (2012). Survival is Success: Journalistic Online start-Ups in Western Europe. Reuters Institute for the Study of Journalism, Universidad de Oxford. ISBN: 978-1-907384-08-0

http://reutersinstitute.politics.ox.ac.uk/sites/default/files/Survival\%20is\%20Success\%20Journalistic\%200nline\%20StartUps\%20in\%20Western\%20Europe_0.pdf

CALVO, I. (2016). Análisis de las disfunciones informativas en la prensa deportiva digital española. Tesis doctoral inédita, Universidad CEU-San Pablo de Madrid. 
CARVAJAL, M. (coord..) (2014). Ranking de innovación periodística 2014. Elche: Universidad Miguel Hernández

http://mip.umh.es/ranking

BRIGGS, M.E. (2012). Entrepreneurial Journalism: How to Build What's Next for News. Sage: Los AngelesLondres

CASERO-RIPOLLÉS, A. (2010). “Prensa en internet: nuevos modelos de negocio en el escenario de la convergencia". El Profesional de la Información, Núm. 6, pp. 595-600

http://www.elprofesionaldelainformacion.com/contenidos/2010/noviembre/05.html

MANFREDI J.L.; ROJAS TORRIJOS, J. L.; y HERRANZ, J.M. (2015). “Periodismo emprendedor: el periodismo deportivo en España". Revista Latina de Comunicación Social, Núm. 70, 2015. Universidad de La Laguna (Tenerife), pp. 69-90

http://www.revistalatinacs.org/070/paper/1035-UC/05es.html

- (2015b). "Innovación en el periodismo emprendedor deportivo. Modelo de negocio y narrativas". EI Profesional de la Información, Núm. 24 (3), pp. 265-273

http://www.elprofesionaldelainformacion.com/contenidos/2015/may/06.html

MANN, N. (2015). “The Great Journalism Innovation Problem”. En Medium:

https://medium.com/@fieldproducer/the-great-journalism-innovation-problemac7ba41cb77c\#.m3q4nojzm

MARTíNEZ CARAZO, P. C. (2006). El método de estudio de caso: estrategia metodológica de investigación científica. Pensamiento y gestión, N²0. ISSN 1657-6276

http://ciruelo.uninorte.edu.co/pdf/pensamiento_gestion/20/5_El_metodo_de_estudio_de_caso.pdf

NEWMAN, N. (2016). Media, Journalism and Technology Predictions 2016. Oxford: Reuters Institute for the Study of Journalism

http://www.digitalnewsreport.org/publications/2016/predictions-2016/

PHILIPS, A. (2015). Journalism in Context. Practice and Theory for the Digital Age. Londres: Routledge ISBN: 978-0-415-53627-1

STAKE, R.E. (1995). Investigación con estudio de caso. Madrid: Ediciones Morata

- (2010). Qualitative Research: studying how things work. The Guilford Press, Nueva York

\section{WEBGRAFÍA}

MARKETINGDIRECTO.COM (2015). La inversión publicitaria crece un 7\% durante el primer semestre de 2015

http://www.marketingdirecto.com/marketing-general/publicidad/la-inversion-publicitaria-crece-un-7durante-el-primer-semestre-de-2015-segun-infoadex/

ROJAS TORRIJOS, J. L., Censo de nuevos medios deportivos lanzados por periodistas en España, 2014

http://periodismodeportivodecalidad.blogspot.com.es/2014/02/censo-de-nuevos-medios-deportivos.html 
(1) Estas herramientas ofrecen la posición de un determinado sitio web en el contexto español, atendiendo a diversos criterios, como el tráfico. Ponderar los datos de ambas permite conocer, de una forma más fiable, cuál es la importancia de un determinado medio digital respecto al resto de proyectos.

(2) La metodología Lean Startup, orientada al lanzamiento de productos y el desarrollo de negocios, se basa en el aprendizaje validado, la experimentación científica y el ensayo-error, con el objetivo de acortar los ciclos de desarrollo, medir el éxito o el fracaso y conseguir feedback de los potenciales clientes. El objetivo, en definitiva, es dedicar únicamente los recursos estrictamente necesarios y modificar la idea de negocio y su ejecución, antes de perder recursos excesivos, en caso de que sea necesario.

\section{BREVE SEMBLANZA DE LOS AUTORES}

José Luis Rojas Torrijos. Profesor Asociado de Periodismo en la Facultad de Comunicación de la Universidad de Sevilla. Doctor en Periodismo, forma parte del Grupo de Investigación Estudio de Medios para un Periodismo de Calidad, del Departamento de Periodismo II de la Universidad de Sevilla.

Cristian Ramón Marín Sanchiz. Graduado en Periodismo por la Universidad Miguel Hernández y estudiante del Máster en Innovación en Periodismo. Investigador especializado en Innovación y Emprendimiento en Periodismo, actualmente desarrolla su carrera profesional en el ámbito de la información y la comunicación relacionada con motociclismo

Ámbitos. Revista Internacional de Comunicación, n.33, edición de verano, 2016.

Recibido: 14/04/2016

Aprobado: 06/06/2016 